\title{
Expression of 4F2he (CD98) in pulmonary neuroendocrine tumors
}

\author{
KYOICHI KAIRA ${ }^{1}$, YASUHISA OHDE ${ }^{4}$, MASAHIRO ENDO ${ }^{2}$, KAZUO NAKAGAWA ${ }^{4}$, \\ TAKEHIRO OKUMURA ${ }^{4}$, TOSHIAKI TAKAHASHI ${ }^{1}$, HARUYASU MURAKAMI $^{1}$, \\ ASUKA TSUYA $^{1}$, YUKIKO NAKAMURA ${ }^{1}$, TATEAKI NAITO ${ }^{1}$, HARUHIKO KONDO ${ }^{4}$, \\ TAKASHI NAKAJIMA $^{3}$ and NOBUYUKI YAMAMOTO ${ }^{1}$ \\ Divisions of ${ }^{1}$ Thoracic Oncology, ${ }^{2}$ Diagnostic Radiology, ${ }^{3}$ Pathology, and ${ }^{4}$ Thoracic Surgery, \\ Shizuoka Cancer Center, 1007 Shimonagakubo Nagaizumi-cho, Sunto-gun, Shizuoka 411-8777, Japan
}

Received May 11, 2011; Accepted June 14, 2011

DOI: $10.3892 / o r .2011 .1384$

\begin{abstract}
F2he (CD98) has been associated with tumor growth, and is highly expressed in various tumors. The aim of this study was to evaluate the clinicopathological significance of $4 \mathrm{~F} 2 \mathrm{hc}$ expression in pulmonary neuroendocrine (NE) tumors. Surgically-resected patient tumors including 16 large cell neuroendocrine carcinoma (LCNEC), 12 small cell lung cancer (SCLC), 1 atypical carcinoid (AC) and 5 typical carcinoid (TC) samples were included in this study. Tumor sections were immunohistochemically stained for 4F2hc (CD98), glucose transporter 1 (Glut1) and 3 (Glut3), hypoxia-inducible factor-1 $\alpha$ (HIF-1 $\alpha$ ), hexokinase I, vascular endothelial growth factor (VEGF), microvessel density (CD34), epidermal growth factor receptor (EGFR), Akt/mammalian target of rapamycin (mTOR) signaling pathway (p-Akt, p-mTOR and p-S6K) and for a cell cycle regulator (p53). 4F2hc was overexpressed in $0 \%$ of the pulmonary carcinoids (TCs and ACs), $62.5 \%$ of the LCNECs and $50.0 \%$ of the SCLCs. A positive 4F2he expression was significantly associated with age, histology and Glut1 expression. Moreover, a significant correlation was found between 4F2hc expression, and Glut1, HIF-1 $\alpha$, p-Akt, p-mTOR and $\mathrm{p}-\mathrm{S} 6 \mathrm{~K}$. The expression of $4 \mathrm{~F} 2 \mathrm{hc}$ was also significantly associated with poor overall survival. The expression of $4 \mathrm{~F} 2 \mathrm{hc}$ expression tended to increase from low-grade to high-grade pulmonary NE tumors. Our results suggest that $4 \mathrm{~F} 2 \mathrm{hc}$ may play a significant role in tumor progression, hypoxic conditions and poor outcome in patients with pulmonary NE tumors.
\end{abstract}

\section{Introduction}

Neuroendocrine (NE) tumors of the lung arise from Kulchitzky cells, which are normally present in the bronchial mucosa and

Correspondence to: Dr Kyoichi Kaira, Division of Thoracic Oncology, Shizuoka Cancer Center, 1007 Shimonagakubo, Nagaizumi-cho, Sunto-gun, Shizuoka 411-8777, Japan

E-mail: kkaira1970@yahoo.co.jp

Key words: pulmonary neuroendocrine tumor, 4F2hc, glucose transporter, hypoxia, mTOR share the common morphological features of neuroendocrine tumors, including organoid nesting, palisading, rosettes, or a trabecular growth pattern. These tumors are represented by a wide range of pathological entities (1-5). It is now widely recognized that NE tumors of the lung include a spectrum that ranges from low-grade typical carcinoid (TC) and intermediate-grade atypical carcinoid (AC) to high-grade large cell neuroendocrine carcinoma (LCNEC) and small cell lung carcinomas (SCLC) (1-4). However, there has been no established clinical marker, which correlates with the response to the treatment and the prognosis in patients with pulmonary NE tumors.

4F2hc (CD98) is a disulphide-linked 125-kDa heterodimeric membrane glycoprotein, which is found on the cell surface of most normal cells. It was first identified on the cell surface of T lymphocytes (5). However, 4F2hc has been shown to be involved in the cellular proliferation, transformation, fusion, and adhesion and also in the L-type amino acid transport (LAT) system, in addition to regulating integrin activation, and therefore, integrin signaling and anchorage-independent growth. 4F2hc is reconstituted and expressed at high levels on the surface of many types of tumor cells. The overexpression of 4F2hc has been shown to result in cellular transformation $(6,7)$. Recent studies have documented that 4F2hc expression is increased in a variety of carcinomas and has a crucial role on the tumor progression and metastasis of human neoplasms (8-14). Recent studies have described that the expression of $4 \mathrm{~F} 2 \mathrm{hc}$ correlates with cell proliferation and angiogenesis, and 4F2hc could be a significant prognostic factor for predicting poor outcome in non-small cell lung cancer (NSCLC) (10). However, little is known about how 4F2hc is associated with the pathogenesis and development of patients with pulmonary NE tumors.

Tumor cells have an increased demand for nutrients such as glucose, amino acids, fatty acids, vitamins and micronutrients. This demand is increased by the availability of nutrients through vascular formation and enhanced by cellular entry of nutrients through up-regulation of specific transporters. Cancer cells enhance glucose uptake via induction of glucose transporter 1 (Glut1) and Glut3, and meet their amino acid demands by induction of the L-type amino acid transporter 1 (LAT1) $(15,16)$. LAT1 requires covalent association with the $4 \mathrm{~F} 2 \mathrm{hc}$ for its functional expression in the plasma membrane 
(16), and a positive LAT1 expression has been described to be correlated with the grade of neuroendocrine tumors of the lung (5). A recent study suggests that 4F2hc stabilizes Glut1 protein and contributes to the regulation not only of amino acids, but also of glucose metabolism (17). Glut1 (and Glut3) is thought to be regulated by hypoxia in a hypoxia-inducible factor-1 (HIF-1)-dependent manner (15). HIF-1 $\alpha$ is one of the factors responsible for the regulation of Glut1 in tumor cells, and is considered to be support tumor growth by the angiogenesis of the expression of vascular endothelial growth factor (VEGF) (15). One in vitro study has documented that not only glucose transporters but also amino acid transporters are subject to regulation in hypoxia (18). HIF-1 $\alpha$ is a downstream component of the mammalian target of rapamycin (mTOR), which is a downstream component of the phosphatidylinositol-3-kinase (PI3K)/ Akt pathway involved in the regulation of cell proliferation, angiogenesis, and amino acid metabolism. However, it remains unknown whether $4 \mathrm{~F} 2 \mathrm{hc}$ is associated with the expression of these hypoxic markers and with the activation of the Akt/mTOR pathway. Therefore, we conducted an immunohistochemical study to examine how 4F2hc expression is correlated with hypoxic markers [Glut1, Glut3, hexokinase I, HIF-1 $\alpha$, VEGF and microvessel density (MVD) determined by CD34] and Akt/ mTOR pathway [epidermal growth factor receptor (EGFR), phospho-Akt, phospho-mTOR and phospho-S6K] and cell cycle regulator (p53) in patients with resected pulmonary NE tumors.

\section{Materials and methods}

Patients. Between March 2003 and October 2009, we analyzed 38 consecutive patients with pulmonary NE tumors who underwent curative resection at the Shizuoka Cancer Center. Of these patients, four patients were excluded from further studies because the tissue specimen was not available. Thus, a total of 34 patients were analyzed in the study. The clinical records of all patients were reviewed to assess the prognosis after surgery. All NE tumors had been diagnosed based on the definitions of the revised WHO classification of lung cancer (19). The study protocol was approved by the institutional review board.

There were 24 male and 10 female patients, and the median age was 70 years (range 51-78 years). Lobectomy had been performed in all patients. The postoperative pathological stage was determined according to the UICC staging system. The pathological diagnoses were: TC $(n=5)$, AC $(n=1)$, SCLC $(n=12)$ and LCNEC $(n=16)$. The median value of maximal tumor size was $26 \mathrm{~mm}$ (range 11-60 mm). Twenty-three patients were of pathological disease stage I and 11 patients were of stage II. Adjuvant chemotherapy had been performed in 11 patients ( 7 patients treated by cisplatin plus etopiside and 4 patients by cisplatin plus irinotecan), and the other patients had received surgery alone. Patients underwent $2-\left[{ }^{18} \mathrm{~F}\right]$-fluoro2-deoxy-D-glucose $\left({ }^{18} \mathrm{~F}\right.$-FDG) positron emission tomography (PET) scanning before curative surgery. The median follow-up period was 24 months; 17 patients were alive, and 17 had died of recurrence. The median progression-free survival (PFS) was 14 months. The median follow-up period of each histological type of NE tumors was as follows: TC and AC (65 months, range 56-85), LCNEC (23 months, range 9-75), and SCLC (21 months, range 6-48).
Immunohistochemical staining. Immunohistochemical staining was performed according to the procedure described in previous reports $(10,15)$. The following antibodies were used: a rabbit polyclonal against Glut1 (AB15309, Abcam, Tokyo, Japan, 1:200 dilution); a rabbit polyclonal against Glut3 (AB15311, Abcam, 1:100 dilution); a rabbit monoclonal against hexokinase I (AB55144, Abcam, 1:200 dilution); a mouse monoclonal against HIF-1 $\alpha$ (NB100-123, Novus Biologicals, Inc., Littleton, CO, 1:50 dilution); a monoclonal against VEGF (Immuno-Biological Laboratories Co., Ltd., Japan, 1:300 dilution); a mouse monoclonal against CD34 (Nichirei, Tokyo, Japan, 1:800 dilution); a mouse monoclonal against EGFR (Novovastra Laboratories Ltd., Newcastle, UK, 1:100 dilution); a rabbit polyclonal against phospho-Akt (Abcam, Tokyo, Japan, 1:200 dilution); a rabbit monoclonal against phospho-mTOR (Cell Signaling, 1:80 dilution); a rabbit monoclonal against phospho-S6K (Cell Signaling, 1:100 dilution) and a mouse monoclonal against p53 (D07; Dako, 1:50 dilution). 4F2hc (CD98) was an affinity purified goat polyclonal antibody (Santa Cruz Biotechnology, Inc., Santa Cruz, USA, 1:200 dilution) raised against a peptide mapping at the carboxy terminus of CD98 of human origin.

The expression of 4F2hc, Glut1, Glut3, EGFR was considered positive if distinct membrane staining was present. Five fields (x400) were analyzed to determine the frequency of the HIF-1 $\alpha$ stained nuclei and hexokinase I stained cytoplasm. For 4F2hc, Glut1, Glut3, EGFR, HIF-1 $\alpha$ and hexokinase I, a semiquantitative scoring method was used: $1,<10 \% ; 2,10-25 \% ; 3$, $25-50 \% ; 4,51-75 \%$; and $5,>75 \%$ of cells positive. The tumors in which stained tumor cells made up $>25 \%$ of the tumor were graded as positive.

The expression of VEGF was quantitatively assessed according to the percentage of immunoreactive cells in a total of 1000 neoplastic cells. The number of CD34-positive vessels was counted in four selected hot spots in a $\mathrm{x} 400$ field $\left(0.26 \mathrm{~mm}^{2}\right.$ field area). MVD was defined as the mean count of microvessels per $0.26 \mathrm{~mm}^{2}$ field area.

p-Akt, p-mTOR and p-S6K were considered positive if membranous and/or cytoplasmic staining was present, and PTEN was positive if nuclear staining. For p-Akt, p-mTOR and p-S6K, a semi-quantitative scoring method was used: $1,<10 \%$; $2,10-25 \% ; 3,25-50 \% ; 4,51-75 \%$; and $5,>75 \%$ of cells positive. The tumors in which stained tumor cells made up $>25 \%$ of the tumor were graded as positive. For p53, microscopic examination for the nuclear reaction product was performed and scored. According to previous report (15), p53 expression in $>10 \%$ of tumor cells was defined as high expression. Sections were assessed using light microscopy in a blinded fashion by at least two of the authors.

Statistical analysis. Probability values of $<0.05$ indicated a statistically significant difference. The Fisher's exact test was used to examine the association of two categorical variables. Correlation of different variables was analyzed using the non-parametric Spearman's rank test. The Kaplan-Meier method was used to estimate survival as a function of time, and the survival differences were analyzed by the log-rank test. Multivariate analyses were performed using stepwise Cox proportional hazards model to identify independent prognostic factors. Statistical analysis was performed using JMP 8 (SAS, Institute Inc., Cary, NC, USA) for Windows. 
A

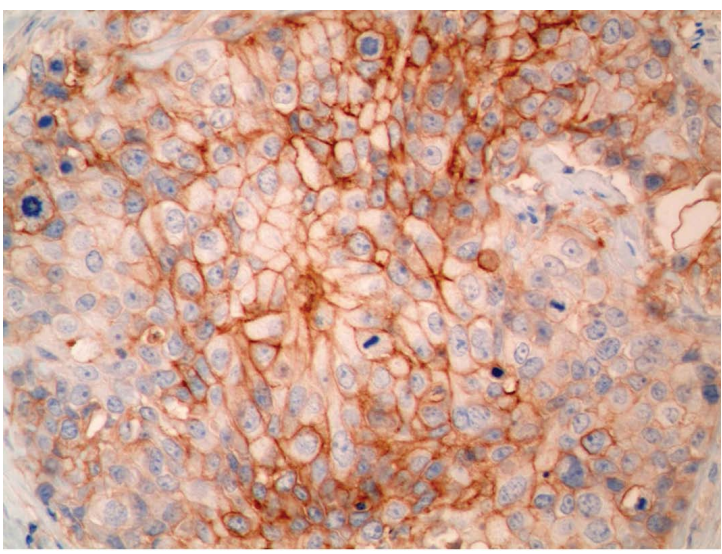

B

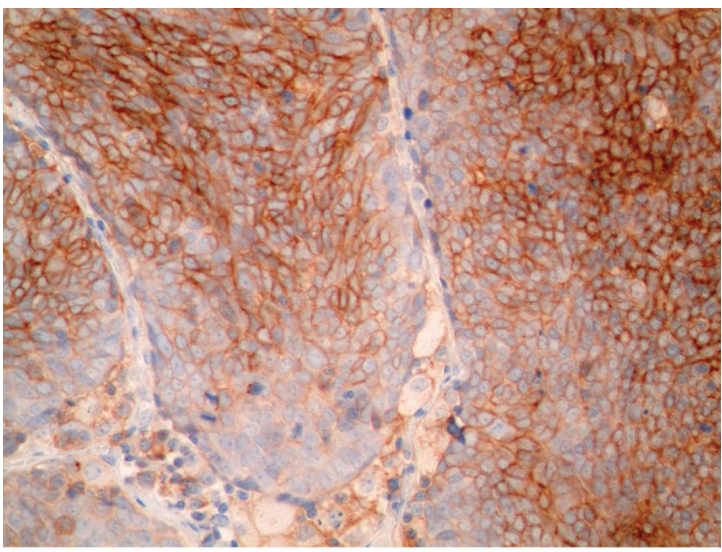

C

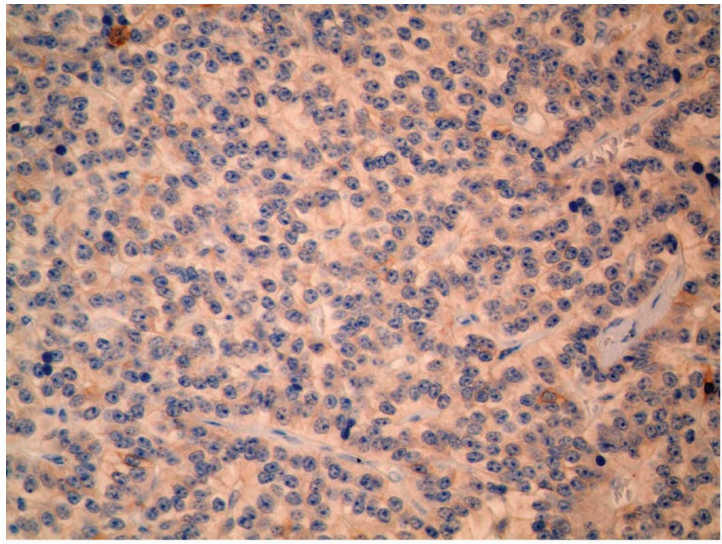

Figure 1. Immunohistochemical expression of 4F2hc in pulmonary neuroendocrine tumors. (A) Positive staining of 4F2hc expression in large cel neuroendocrine carcinoma. The score of $4 \mathrm{~F} 2 \mathrm{hc}$ immunostaining was grade 4, and its immunohistostaining pattern was membranous. (B) 4F2hc expression in small cell lung cancer with a staining score of grade 3. (C) Negative 4F2hc staining in a typical carcinoid.

\section{Results}

Immunohistochemical analysis. 4F2hc immunostaining was detected in carcinoma cells in tumor tissues and localized predominantly on their plasma membrane (Fig. 1). The cytoplasmic staining was rarely evident. In the present study, no expression of $4 \mathrm{~F} 2 \mathrm{hc}$ protein was observed in any normal epithelial cells of the lung, including bronchial epithelial and alveolar cells. A positive expression was recognized in $0 \%$ $(0 / 6)$ of pulmonary carcinoid (TC and AC), 62.5\% (10/16) of LCNEC and 50\% (6/12) of SCLC, respectively. The results of
Table I. Immunohistochemistry for $4 \mathrm{~F} 2 \mathrm{hc}$ in pulmonary neuroendocrine tumors.

\begin{tabular}{lrrrrr}
\hline & & \multicolumn{4}{c}{ Score } \\
\cline { 3 - 6 } Diagnosis & $\mathrm{n}$ & 1 & 2 & 3 & 4 \\
\hline TC & 5 & 5 & 0 & 0 & 0 \\
AC & 1 & 0 & 1 & 0 & 0 \\
LCNEC & 16 & 2 & 4 & 8 & 2 \\
SCLC & 12 & 2 & 4 & 6 & 0 \\
\hline
\end{tabular}

TC, typical carcinoid; AC, atypical carcinoid; LCNEC, large cell neuroendocrine carcinoma; SCLC, small cell lung cancer.

the immunohistochemical analysis of $4 \mathrm{~F} 2 \mathrm{hc}$ protein expression in pulmonary NR tumors are summarized in Table I. In a quantitative analysis, the mean scoring of $4 \mathrm{~F} 2 \mathrm{hc}$ expression was significantly higher in LCNEC $(p=0.014)$ and SCLC $(\mathrm{p}=0.014)$ than in pulmonary carcinoid, demonstrating no significant difference between LCNEC and SCLC ( $p=0.091)$.

Glut 1 and Glut 3 were detected in tumor cells and localized predominantly on their plasma membrane. A positive rate of Glut 1 and Glut 3 expression was observed in $79.4 \%$ (27/34) and $11.7 \%(4 / 34)$ of the tumors, respectively. A positive expression of HIF-1 $\alpha$ was predominantly observed in the cytoplasm with some nuclear staining, and was observed in $85.2 \%(29 / 34)$ of the tumors. A positive expression of hexokinase I was observed in the cytoplasm and/or membrane of neoplastic cells, and was observed in 58.8\% (20/34) of the tumors. The median rate of VEGF positivity was $11.0 \%$ (range, 1-60\%), and the median percent of CD34 positivity was 28 (range, $4-54 \%$ ). The positive rates of VEGF and CD34 were $44.1 \%(15 / 34)$ and $41.1 \%$ (14/34), respectively. A positive expression of EGFR, p-AKT, p-mTOR and p-S6K was detected in $70.5 \%(24 / 34)$, $26.4 \%(9 / 34), 29.4 \%(10 / 34)$ and $32.3 \%$ (11/34) of the tumors, respectively.

The positive rate of these biomarkers according to histological types is shown in Fig. 2A. The positive rate of Glut1 expression was significantly higher in LCNEC $(\mathrm{p}=0.0043)$ and SCLC ( $p=0.0039)$ than pulmonary carcinoid, demonstrating no significant difference between LCNEC and SCLC. The positive expression of HIF-1 $\alpha$ was significantly higher in SCLC than in pulmonary carcinoid $(\mathrm{p}=0.0245)$, demonstrating no significant difference between LCNEC and pulmonary carcinoid. No statistically significant difference in the positive expression of the other biomarkers was recognized among these histological types (pulmonary carcinoid, LCNEC and SCLC).

Relationship between $4 F 2 h c$ and different variables. The results of the relationship between 4F2hc expression and different variables are listed in Table II. A positive 4F2hc expression was significantly associated with age, histology and Glut1. Fig. 2 represents the comparison between 4F2hc positive and negative expression according to the positive rate of different biomarkers. Using the Spearman rank correlation, the expression of $4 \mathrm{~F} 2 \mathrm{hc}$ was significantly correlated with Glut1, HIF-1 $\alpha$, p-Akt, p-mTOR and p-S6K (Table III). 
A

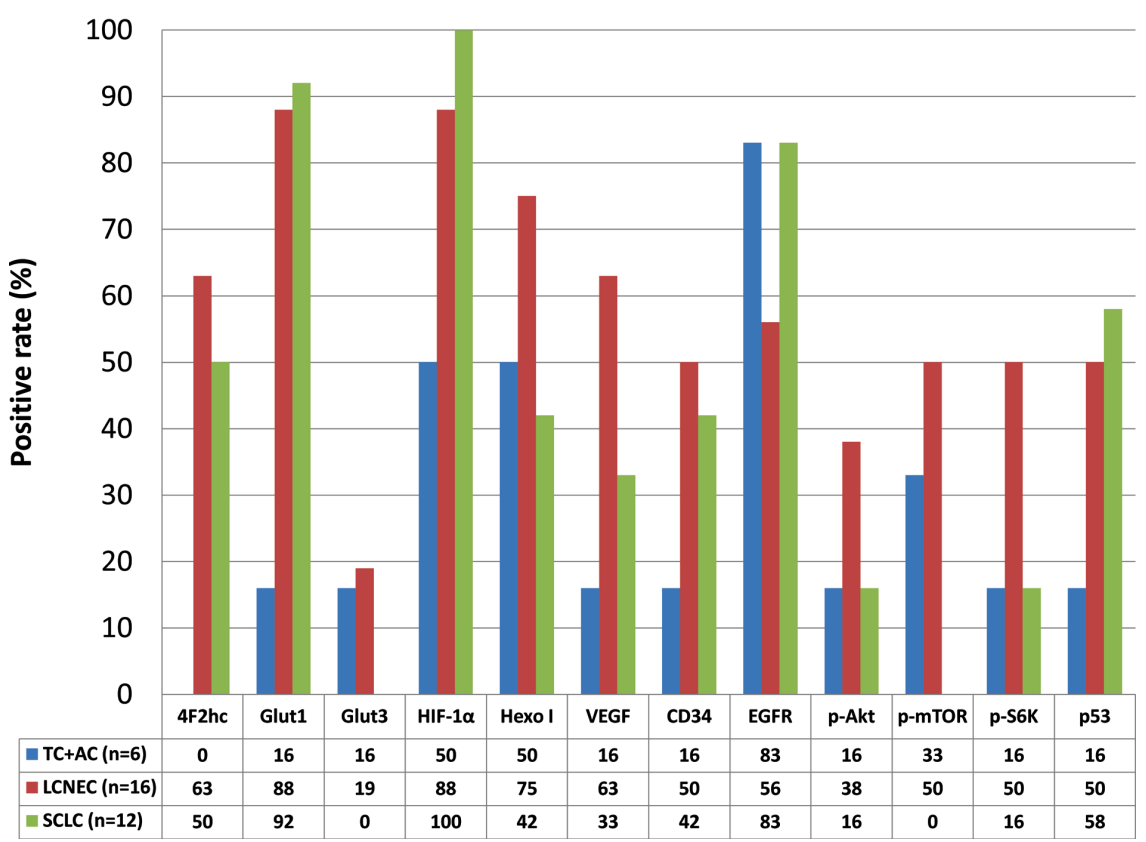

B

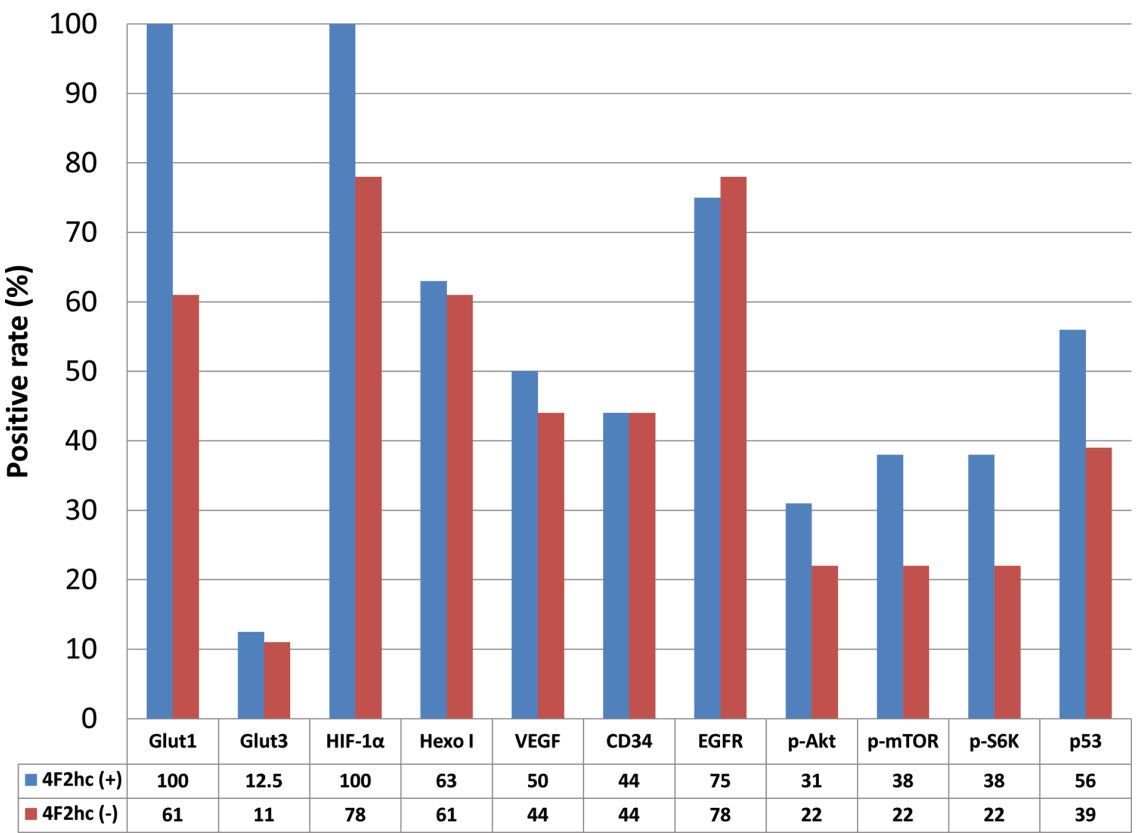

Figure 2. (A) Comparison of the positive rate of these biomarkers according to histological types. Positive rate of Glut1 expression was significantly higher in LCNEC ( $\mathrm{p}=0.0043$ ) and SCLC ( $\mathrm{p}=0.0039)$ than pulmonary carcinoid (TC and AC), demonstrating no significant difference between LCNEC and SCLC. Positive expression of HIF-1 $\alpha$ was significantly higher in SCLC than pulmonary carcinoid $(p=0.0245)$, demonstrating no significant difference between LCNEC and pulmonary carcinoid. No statistically significant difference in the positive expression of the other biomarkers was recognized among pulmonary carcinoid, LCNEC and SCLC. (B) Comparison of the positive rate of different biomarkers according to 4F2hc expression. A statistically significant difference in the expression of Glut1 was observed between $4 \mathrm{~F} 2 \mathrm{hc}$ positive and negative patients, demonstrating no significant difference in the other biomarkers.

Survival analysis. The 5-year survival rate and median survival time (MST) was $41.9 \%$ and 54 months, respectively. The 5-year survival rate and MST of each histological type of the NE tumors was as follows: pulmonary carcinoid $(100 \%$, not reached), LCNEC (47.8\%, 54 months), and SCLC (0\%, 26 months). Table IV shows the results of univariate and multivariate analysis in the overall survival (OS) according to the different variables. Univariate analysis demonstrated that gender, disease stage, histology, 4F2hc and Glut1 were significantly associated with poor overall survival. Survival curves by the Kaplan-Meier method also showed a statistically significant separation of 4F2hc-positive vs. 4F2hc-negative patients for all neuroendocrine carcinomas $(p=0.042)$ (Fig. 3A), but not for high-grade neuroendocrine carcinomas $(\mathrm{p}=0.590)$ (Fig. 3B). According to the results of the univariate log-rank test in all patients $(n=34)$, we screened the prognostic factors with a cut-off of $\mathrm{p}<0.05$; those included gender, disease stage, histology, 4F2hc and Glut1. Multivariate analysis 
Table II. Relationship between 4F2hc expression and different variables.

\begin{tabular}{|c|c|c|c|}
\hline Variables & $\begin{array}{l}\text { 4F } 2 \mathrm{hc}(+) \\
\quad(\mathrm{n}=16)\end{array}$ & $\begin{array}{l}\text { 4F2hc }(-) \\
(\mathrm{n}=18)\end{array}$ & p-value \\
\hline \multicolumn{4}{|l|}{ Age (years) } \\
\hline$\leq 65 />65$ & $3 / 13$ & $12 / 6$ & 0.007 \\
\hline \multicolumn{4}{|l|}{ Gender } \\
\hline Male/female & $12 / 4$ & $12 / 6$ & 0.714 \\
\hline \multicolumn{4}{|l|}{ Pathological stage } \\
\hline $\mathrm{I} / \mathrm{II}$ & $9 / 7$ & $14 / 4$ & 0.274 \\
\hline \multicolumn{4}{|l|}{ Maximal size of tumor } \\
\hline $\begin{array}{l}\text { Histological feature } \\
(\mathrm{TC}+\mathrm{AC}) /(\mathrm{SCLC}+\mathrm{LCNEC})\end{array}$ & $0 / 16$ & $6 / 12$ & 0.019 \\
\hline \multicolumn{4}{|l|}{ Pleural involvement } \\
\hline \multicolumn{4}{|l|}{ Vascular invasion } \\
\hline $\begin{array}{l}\text { Lymphatic permeation } \\
\text { Positive/negative }\end{array}$ & $5 / 11$ & $9 / 9$ & 0.315 \\
\hline \multicolumn{4}{|l|}{ Glut1 } \\
\hline \multicolumn{4}{|l|}{ Glut3 } \\
\hline \multicolumn{4}{|l|}{ Hexokinase I } \\
\hline \multicolumn{4}{|l|}{ HIF- $1 \alpha$} \\
\hline \multicolumn{4}{|l|}{ VEGF } \\
\hline \multicolumn{4}{|l|}{ CD34 } \\
\hline \multicolumn{4}{|l|}{ EGFR } \\
\hline \multicolumn{4}{|l|}{ p-Akt } \\
\hline Positive/negative & $5 / 11$ & $4 / 14$ & 0.702 \\
\hline \multicolumn{4}{|l|}{ p-mTOR } \\
\hline \multicolumn{4}{|l|}{ p-S6K } \\
\hline Positive/negative & $6 / 10$ & $4 / 14$ & 0.456 \\
\hline \multicolumn{4}{|l|}{$\mathrm{p} 53$} \\
\hline Positive/negative & $9 / 7$ & $7 / 11$ & 0.492 \\
\hline
\end{tabular}

Glut1, glucose transporter 1; Glut3, glucose transporter 3; HIF-1 $\alpha$, hypoxia inducible factor-1 $\alpha$; VEGF, vascular endothelial growth factor; EGFR, epidermal growth factor receptor; mTOR, mammalian target of rapamycin; TC, typical carcinoid; AC, atypical carcinoid; LCNEC, large cell neuroendocrine carcinoma; SCLC, small cell lung cancer.

demonstrated that histology was an independent prognostic factor for predicting poor outcome.
Table III. Relationship between 4F2hc expression and biomarkers.

\begin{tabular}{|c|c|c|c|}
\hline Biomarkers & Spearman $\gamma$ & $\begin{array}{c}95 \% \text { Confidence } \\
\text { interval }\end{array}$ & p-value \\
\hline Glut1 & 0.537 & $0.233-0.745$ & 0.001 \\
\hline Glut3 & 0.299 & $-0.053-0.585$ & 0.085 \\
\hline Hexokinase I & 0.218 & $-0.139-0.526$ & 0.214 \\
\hline $\mathrm{HIF}-1 \alpha$ & 0.355 & $0.009-0.625$ & 0.039 \\
\hline VEGF & 0.284 & $-0.069-0.575$ & 0.120 \\
\hline CD34 & 0.208 & $-0.149-0.518$ & 0.208 \\
\hline EFGR & 0.100 & $-0.256-0.432$ & 0.572 \\
\hline p-Akt & 0.438 & $0.107-0.682$ & 0.009 \\
\hline p-mTOR & 0.344 & $-0.003-0.617$ & 0.046 \\
\hline p-S6K & 0.445 & $0.116-0.686$ & 0.008 \\
\hline
\end{tabular}

Glut1, glucose transporter 1; Glut3, glucose transporter 3; HIF-1 $\alpha$, hypoxia inducible factor-1 $\alpha$; VEGF, vascular endothelial growth factor; EGFR, epidermal growth factor receptor; mTOR, mammalian target of rapamycin.
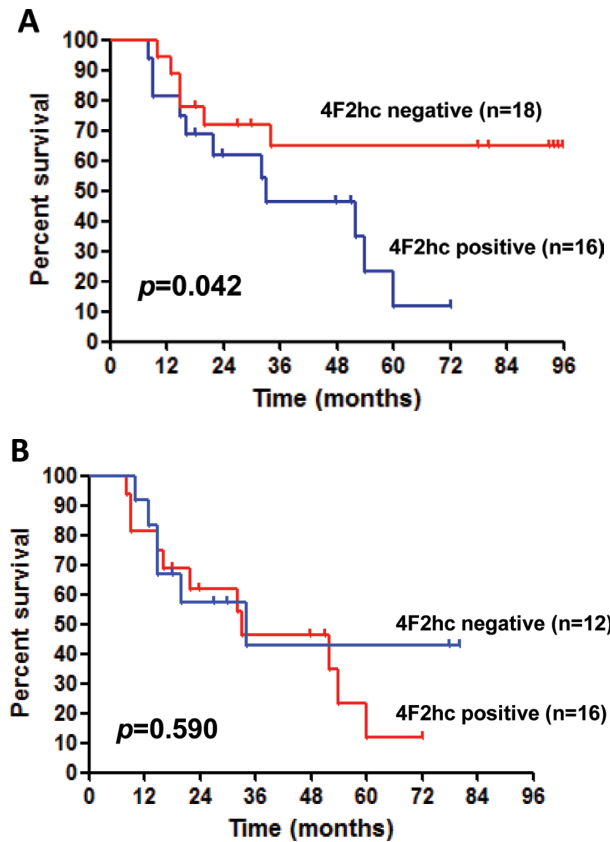

Figure 3. Kaplan-Meier survival analysis for $4 \mathrm{~F} 2 \mathrm{hc}$ expression in all patients $(n=34)$ (A) and high-grade NE tumor (LCNEC and SCLC; $n=28$ ). Difference in overall survival between subgroups was analyzed using log-rank test.

\section{Discussion}

This is the first report of a clinicopathological study designed to investigate the expression of $4 \mathrm{~F} 2 \mathrm{hc}$ in pulmonary NE tumors. In the present study, 4F $2 \mathrm{hc}$ was overexpressed in $0 \%$ of the pulmonary carcinoid, $62.5 \%$ of the LCNEC and $50.0 \%$ of the SCLC. A positive 4F2hc expression was significantly associated with age, histology and Glut1. Moreover, a significant correlation was found between 4F2hc expression, and Glut1, HIF-1 $\alpha$, p-Akt, p-mTOR and p-S6K. 4F2hc was also signifi- 
Table IV. Univariate and multivariate analysis of the overall survival.

\begin{tabular}{cccc}
\hline & $\begin{array}{c}\text { Univariate } \\
\text { analysis }\end{array}$ & & $\begin{array}{c}\text { Multivariate } \\
\text { analysis }\end{array}$ \\
\cline { 2 - 2 } Variables & 5 -year rate (\%) & $\mathrm{p}$-value & $\mathrm{p}$-value \\
\hline
\end{tabular}

Age (years)

$\leq 65 />65$

$57.1 / 48.1$

0.618

Gender

Male/female

$37.8 / 78.7$

0.049

0.175

Stage

I/II

$62.1 / 23.3$

0.014

0.051

Histology

$(\mathrm{TC}+\mathrm{AC}) /(\mathrm{LCNEC}+\mathrm{SCLC}) \quad$ 100/35.5

0.016

0.025

$4 \mathrm{~F} 2 \mathrm{hc}$

Positive/negative

$11.6 / 64.6$

0.042

0.163

Glut1

Positive/negative

$32.8 / 87.5$

0.023

0.207

Glut3

Positive/negative

$66.6 / 48.9$

HIF-1 $\alpha$

Positive/negative

$43.4 / 80.0$

0.199

Hexokinase I

Positive/negative

$46.1 / 56.2$

0.775

VEGF

Positive/negative

28.6/57.0

CD34

Positive/negative

28.1/57.5

0.437

EGFR

Positive/negative

$59.4 / 34.1$

0.192

p-Akt

Positive/negative

$37.0 / 56.0$

0.688

p-mTOR

Positive/negative

68.5/43.0

0.332

p-S6K

Positive/negative

49.1/50.1

0.978

p53

Positive/negative

$55.6 / 49.7$

0.721

Glut, glucose transporter; HIF-1 $\alpha$, hypoxia inducible factor-1 $\alpha$; VEGF, vascular endothelial growth factor; EGFR, epidermal growth factor receptor; mTOR, mammalian target of rapamycin; MST, median survival time (month); NR, not reached; TC, typical carcinoid; AC, atypical carcinoid; LCNEC, large cell neuroendocrine carcinoma; SCLC, small cell lung cancer; 5-year rate, 5-year survival rate.

cantly associated with poor OS. We found that the expression of $4 \mathrm{~F} 2 \mathrm{hc}$ tended to increase from low-grade to high-grade NE tumors, and had an essential role on the development and tumor progression of pulmonary NE tumors.

In the present study, $4 \mathrm{~F} 2 \mathrm{hc}$ expression was closely correlated with the expression of Glut1. Ohno et al described that overexpression of $4 \mathrm{~F} 2 \mathrm{hc}$ increased the amount of Glut1 protein with increased glucose uptake in vitro, whereas, siRNA-mediated $4 \mathrm{~F} 2 \mathrm{hc}$ gene suppression markedly reduced Glut1 protein (17). Although the previous reports have already described the close association between LAT1 and 4F2hc expression (10-13), it remains unclear whether $4 \mathrm{~F} 2 \mathrm{hc}$ is closely correlated with Glut1 expression. Our results suggest that $4 \mathrm{~F} 2 \mathrm{hc}$ is associated with the regulation of Glut1 expression in human neoplasms. In the present study, Glut1 expression was also useful for predicting the grade of malignancy and outcome, whereas Glut3 expression was not associated with these factors. Glut1 was highly expressed in patients with LCNEC and SCLC, but Glut 3 expression was low in pulmonary NE tumors. Ozbudak et al has documented that Glut1 expression was observed in 7\% of TC, 74\% of LCNEC and 78\% of SCLC, and significantly correlated with neuroendocrine differentiation/tumor type and poor prognosis (20). In addition, a statistically significant difference in the prognosis according to Glut1 expression was seen for stage I-II $(\mathrm{p}<0.001)$ but not stage III-IV $(p=0.4)$ neuroendocrine carcinomas. Our study also indicated that Glut1 expression was observed in $16 \%$ of pulmonary carcinoid, $88 \%$ of LCNEC and $92 \%$ of SCLC, and was closely related to poor outcome in patients with stage I-II NE tumors. The expression of Glutl also seemed to tend to increase from low-grade to high-grade NE tumors. Moreover, HIF-1 $\alpha$ is responsible for the regulation of Glut 1 expression and is closely correlated with $4 \mathrm{~F} 2 \mathrm{hc}$ expression. Considering that HIF- $1 \alpha$ is closely related to the regulation of Glut $1,4 \mathrm{~F} 2 \mathrm{hc}$ has a potential to play an important role in the development of hypoxia and glucose metabolism in human cancers.

Amino acid nutrition in mammalian cells is coupled to cell signaling via mTOR and coordinates the signal with cell growth and cell cycle progression $(21,22)$. In in vitro studies, the inhibition of LAT1 has been documented to reduce the level of phosphorylation of mTOR and p70S6K, and the results of these reports indicate the close relationship between LAT1 and the mTOR pathway $(21,22)$. In our study, $4 \mathrm{~F} 2 \mathrm{hc}$ was also closely correlated with the activation of the mTOR pathway and the expression of p-Akt, p-mTOR and p-S6K tended to be higher in patients with a positive 4F2hc expression than those with a negative $4 \mathrm{~F} 2 \mathrm{hc}$ expression. The mTOR pathway is an upstream component of hypoxic markers (Glut1 and HIF-1 $\alpha$ ) and these hypoxic markers enhance the expression of $4 \mathrm{~F} 2 \mathrm{hc}$. Thus, the overexpression of $4 \mathrm{~F} 2 \mathrm{hc}$ may contribute to activation of the Akt/mTOR signaling pathway. However, little is known about the relationship between $4 \mathrm{~F} 2 \mathrm{hc}$ expression, hypoxia and the mTOR pathway in pulmonary NE tumors. Therefore, further investigation is warranted.

Recently, 4F2hc has been documented to be an independent prognostic factor for predicting poor outcome in thoracic neoplasms such as NSCLC and thymic epithelial tumors $(10,23)$. In these studies, angiogenesis has an essential role on the development of 4F2hc expression, but it remains unknown how hypoxia and glucose metabolism are associated with the expression of $4 \mathrm{~F} 2 \mathrm{hc}$. In the present study, VEGF and MVD were not related to the overexpression of $4 \mathrm{~F} 2 \mathrm{hc}$. The reasons the results of our study are contradictory to previous reports may be that our study population consisted of patients with early-stage NE tumors (stage I or II) and that our sample size was small. A large-scale study is warranted for verifying our 
results. In addition, our preliminary data also indicated that the expression of $4 \mathrm{~F} 2 \mathrm{hc}$ is useful for predicting poor outcome in all patients with pulmonary NE tumors, but not in those with high-grade NE tumors (LCNEC and ACLC). A previous report described that a statistically significant difference in the OS was observed between Glut1-positive and Glut1-negative patients for all pulmonary neuroendocrine tumors, but not for high-grade neuroendocrine tumors (17). These results suggest that the prognostic significance of 4F2hc expression corresponds to that of Glut1 expression in pulmonary NE tumors, and $4 \mathrm{~F} 2 \mathrm{hc}$ expression has an essential role on the regulation of not only LAT1 but also Glut1.

The present study has several limitations. Firstly, our study population was small and included a heterogeneous group of tumors. Pulmonary NE tumors excluding SCLC are rare neoplasms, therefore, the present study warrants a larger, multicenter cohort study. Another limitation is that only cases with surgical treatment were collected to conduct an immunohistochemical study. In general, most patients with SCLC are not candidates for surgical resection because of local or systemic spread of the tumor. In the present study, the selected cases with SCLC may not represent typical SCLC, which might have an aggressive nature.

In conclusion, 4F2hc expression was observed in LCNEC and SCLC. The frequency of 4F2hc expression tended to increase from low-grade to high-grade NE tumors. 4F2hc expression was significantly correlated with the expression of hypoxic markers and the activation of the Akt/mTOR pathway, and has a close association with poor outcome. Although 4F2hc expression may increase with the up-regulation of a hypoxic condition, metabolic activity and mTOR pathway in pulmonary NE tumors, the mechanism of 4F2hc expression in NE tumors is still unknown. Further studies are warranted for verifying the clinical significance of 4F2hc expression in the therapeutic response and outcome of human cancers. Inhibiting 4F2hc function may cause a cessation of the growth of the tumor and provide new and effective therapeutic target of pulmonary neuroendocrine tumors.

\section{Acknowledgements}

This study was supported in part by Grant 21790793 (K.K) from the Ministry of Education, Culture, Sports, Science and Technology, Japan, and the National Hospital Organization Policy Based Medical Services. We thank all staff of the Pathology Department of the Shizuoka Cancer Center for their technical assistance of immunohistochemical analysis.

\section{References}

1. Cooper WA, Thourani VH, Gal AA, Lee RB, Mansour KA and Miller JI: The surgical spectrum of pulmonary neuroendocrine neoplasms. Chest 119: 14-18, 2001.

2. Travis WD, Linnoila RI, Tsokos MG, et al: Neuroendocrine tumors of the lung with proposed criteria for large-cell neuroendocrine carcinoma: an ultrastructural, immunohistochemical, and flow cytometric study of 35 cases. Am J Surg Pathol 15: 529-553, 1991.
3. Travis WD, Rush W, Flieder DB, et al: Survival analysis of 200 pulmonary neuroendocrine tumors with clarification of criteria for atypical carcinoid and its separation from typical carcinoid. Am J Surg Pathol 22: 934-944, 1998.

4. Asamura H, Kameya T, Matsuno Y, et al: Neuroendocrine neoplasms of the lung: a prognostic spectrum. J Clin Oncol 24 : 70-76, 2006.

5. Kaira K, Oriuchi N, Imai H, et al: Expression of L-type amino acid transporter 1 (LAT1) in neuroendocrine tumors of the lung. Pathol Res Pract 204: 553-561, 2008.

6. Haynes BF, Hemler ME, Mann DL, et al: Characterization of a monoclonal antibody (4F2) that binds to human monocytes and to a subset of activated lymphocytes. J Immunol 126: 1409-1414, 1981.

7. Rintoul RC, Buttery RC, Mackinnon AC, et al: Cross-linking CD98 promotes integrin-like signaling and anchorage-independent growth. Mol Biol Cell 13: 2841-2852, 2002.

8. Takeuchi H, Kubota T, Kitai R, Nakagawa T and Hashimoto N: CD98 immunoreactivity in multinucleated giant cells of glioblastomas: an immunohistochemical double labeling study. Neuropathology 28: 127-131, 2008.

9. Kaira K, Oriuchi N, Shimizu K, et al: Correlation of angiogenesis with ${ }^{18}$ F-FMT and ${ }^{18} \mathrm{~F}$-FDG uptake in non-small cell lung cancer. Cancer Sci 100: 753-758, 2009.

10. Kaira K, Oriuchi N, Imai H, et al: CD98 expression is associated with poor prognosis in resected non-small cell lung cancer with lymph node metastasis. Ann Surg Oncol 16: 3437-3481, 2009.

11. Kaira K, Oriuchi N, Imai H, et al: L-type amino acid transporter 1 (LAT1) and CD98 expression in the primary site and the metastatic site of human neoplasms. Cancer Sci 99: 3280-3286, 2008.

12. Kaira K, Oriuchi N, Imai H, et al: Prognostic significance of L-type amino acid transporter 1 (LAT1) and 4F2 heavy chain (CD98) expression in stage I pulmonary adenocarcinoma. Lung Cancer 66: 120-126, 2009.

13. Kaira K, Oriuchi N, Imai H, et al: Prognostic significance of L-type amino acid transporter 1 (LAT1) and 4F2 heavy chain (CD98) expression in early stage squamous cell carcinoma of the lung. Cancer Sci 100: 249-254, 2009.

14. Kaira K, Oriuchi N, Shimizu K, et al: Evaluation of thoracic tumors with ${ }^{18} \mathrm{~F}$-FMT and ${ }^{18} \mathrm{~F}$-FDG PET-CT: a clinicopathological study. Int J Cancer 124: 1152-1160, 2008.

15. Kaira K,Endo M,Abe M, et al: Biologic correlation of 2- $\left[{ }^{18} \mathrm{~F}\right]$-fluoro2-deoxy-D-glucose uptake on positron emission tomography in thymic epithelial tumors. J Clin Oncol 28: 3746-3753, 2010.

16. Kanai $\mathrm{Y}$, Segawa $\mathrm{H}$, Miyamoto $\mathrm{K}$, et al: Expression cloning and characterization of a transporter for large neutral amino acids activated by the heavy chain of 4F2 antigen (CD98). J Biol Chem 273: 23629-23632, 1998.

17. Ohno H, Nakatsu Y, Sakoda H, et al: 4F2hc stabilizes GLUT1 protein and increases glucose transport activity. Am J Physiol Cell Physiol 300: C1047-C1054, 2011.

18. Soado RJ, Li JY, Tsukamoto H and Pardridge WM: Hypoxia induces de-stabilization of the LAT1 large neural amino acid transporter mRNA in brain capillary endothelial cells. J Neurochem 85: 1037-1042, 2003.

19. Travis WD, Colby TV, Corrin B, Shimosato YA and Brambilla E; In Collaboration with Sobin $\mathrm{LH}$ and Pathologists from 14 Countries. World Health Organization International Histological Classification of Tumours. Histological Typing of Lung and Pleural Tumours. 3rd edition. Springer-Verlag, 1999.

20. Ozbudak IH, Shilo K, Tavora F, et al: Glucose transporter-1 in pulmonary neuroendocrine carcinomas: expression and survival analysis. Modern Pathol 22: 633-638, 2009.

21. Yamauchi K, Sakurai H, Kimura T, et al: System L amino acid transporter inhibitor enhances anti-tumor activity of cisplatin in a head and neck squamous cell carcinoma cell line. Cancer Lett 276: 95-101, 2009.

22. Imai H, Kaira K, Oriuchi N, et al: Inhibition of L-type amino acid transporter 1 has antitumor activity in non-small cell lung cancer. Anticancer Res 30: 4819-4828, 2010.

23. Kaira K, Takahashi T, Abe M, et al: CD98 expression is associated with the grade of malignancy in thymic epithelial tumors. Oncol Rep 24: 861-867, 2010. 